\title{
Novel Passive Optical Switching Using Shared Electrical Buffer and Wavelength Converter
}

\author{
Ji-Hwan Kim ${ }^{1}$, JungYul Choi ${ }^{2}$, Jinsung $\operatorname{Im}^{1}$, Minho Kang ${ }^{1}$, and J.-K. Kevin Rhee ${ }^{1 *}$ \\ ${ }^{1}$ Optical Internet Research Center, ICU 119 Munjiro, Yuseong-Gu, Daejeon, 305-732, \\ South Korea \\ ${ }^{2}$ BcN Business Unit, KT Corp., 463-1 Jeonmin-Dong, Yuseong-Gu, Daejeon, 305-811, \\ South Korea \\ *rhee.jk@ieee.org
}

\begin{abstract}
We propose a novel optical switching system using fast time-slotted passive switching with shared OEO buffers. Simulation results show that the proposed system significantly reduces the blocking probability below $10^{-6}$ at a $28 \%$ cost of a traditional system due to flexibility of contention resolution using electrical buffer and potentially requires very low power consumption. The proposed switching system is believed to be a technoeconomically feasible and implementable solution for optical packet and burst switching with current optical technologies.
\end{abstract}

Keywords: Active buffer, time-slotted switching, passive switch, cost analysis, optical packet/burst switching.

\section{Introduction}

In the next decade with fully featured multimedia network service requirement, the internet will revolve with newer technologies in order to provision the explosive growth of the capacity demand and delicate network QoS and traffic engineering functions. A single domain of a network may deal with petabit per second traffic demands. In order to support this trend, a disruptive technology employment such as optical packet switching (OPS) [1] and optical burst switching (OBS) [2] is considered. However, few technologies in this avenue yet look promising to achieve the industry goals to provide an ultimate bandwidth solution with practical application requirements of performance, cost, power consumption, and form factor. In order to re-investigate the fundamental understandings of the traditional cost-performance optimization problem of optical switch networks, we revisit the old but fundamental themes how to implement buffers in packet switched networks. In addition, because of the special WDM network constraints, the wavelength continuity requirement is also re-investigated. As a result, this paper proposes a novel optical switching system consisting of a passive switch with an active buffer module, which may open a new design concept of an optically transparent network system design. 
The OPS/OBS systems are mainly composed to two essential hardware functions, including the switch fabric and the buffer. The functions may utilize passive and active technologies as shown in Table 1. Most of active technologies utilize semiconductor optical amplifiers (SOAs) and wavelength converters (WCs) that offers capability of rather complicated optical signal processing, at the price of power for high data rates and complexity of control, and premium device technologies. Passive technologies, instead, can mitigate high power requirements and control complexity by far, but not capable of wavelength conversion that is important for providing wavelength continuity in WDM network systems and reducing high packet loss probability.

As one can notice from Table 1, the traditional switch architecture is 'active switches with passive buffers system,' where the wavelength continuity is fully provided [3] by a switch fabric. This design rule, however, may have been the critical road block against achieving low cost, low power, and low blocking probability $(B P)$. This paper reports that 'passive switches with active buffers system' is more effective in the respect of packet loss and overall system cost. Being capable of achieving extraordinary performance, the active buffers can be shared, which can reduce the system cost, power consumption, and form factor dramatically to achieve petabit switch solutions

Table 1. Technology classification for optical switching. Bold-faced technologies suggest possible device solutions for the proposed node architecture design.

\begin{tabular}{|c||c|c|}
\hline Technology & optical switch fabric & optical buffer \\
\hline \hline $\begin{array}{c}\text { Passive } \\
\text { (no WC) }\end{array}$ & $\begin{array}{c}\text { electro-optic, acouto-optic, } \\
\text { ultra-fast MEMS, } \\
\text { wavelength-selective switch }\end{array}$ & $\begin{array}{c}\text { fiber delay line, recirculating loop, } \\
\text { slow-light photonic crystal }\end{array}$ \\
\hline $\begin{array}{c}\text { Active } \\
\text { (WC capable) }\end{array}$ & $\begin{array}{c}\text { electrical switch with OEO, } \\
\text { AWGR/WC, SOA array }\end{array}$ & $\begin{array}{c}\text { electrical memory with OEO, } \\
\text { bistable laser diode }\end{array}$ \\
\hline
\end{tabular}

\section{Passive switching with shared active buffering}

Packet (or burst) switched DWDM networks require packet (or data burst) forwarding to the destined output fiber of a node with or without conversion of the wavelength. When only a fast passive switch fabric is used, the availability of the output fiber is limited to that of Aloha network because of no wavelength conversion. By the theory, several tens of percentiles of the input packets/bursts are lost due to contention. Only the contended packets are then sent to electrical buffers by $\mathrm{O} / \mathrm{E}$ conversion (Fig.1).

As soon as any wavelength channel becomes available at the destined output fiber, the electrical data is converted to the available wavelength by a tunable $\mathrm{E} / \mathrm{O}$ converter, i.e. a tunable transmitter. Because an electrical buffer provides buffering time flexibility and large buffer depth at low costs, $B P$ can be reduced below $10^{-6}$ with a minimal investment of buffers (Fig.2). Here we define the sharing ratio, $S R$, as the ratio of the required number of buffers $B$ to the total number of channels that is the product of the number of wavelength $W$ and the number of fiber ports $F$. 


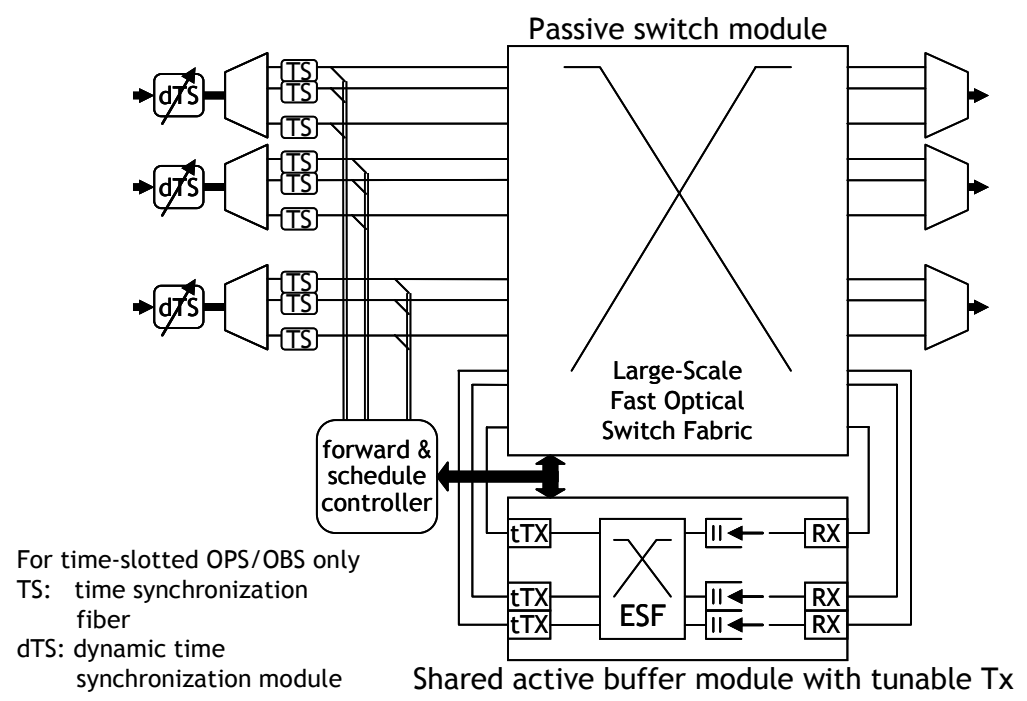

Fig. 1. Schematic node architecture of passive switching and shared active buffering

Time-slotted OPS/OBS [1] in a synchronized network can reduce $B P$ performance of passive-only switching approximate by half in principle, reducing requirement of buffer $S R$ by half or so, leading to an extreme savings of cost, power, and form-factor, as the OEO and buffer are the most cost critical subsystem, as compared in Fig.2. The combination of time-slotted packet switching and passive switching with shared active buffering (PSSAB) can achieve extremely high performance of $B P<10^{-6}$ with tunable OEO buffer sharing ratio of only $15 \%$. This offers major reduction of cost and power consumption because OEOs and WCs are most expensive and powerconsuming part of the system.

The fundamental difference of the proposed idea is that the traditional method of wavelength switch with shared passive fiber-delay-line buffers (WSSPB) [3,4] attempt to resolve contention by wavelength conversion and the rest of contention is resolved by shallow buffering, while our scheme uses deep buffering first and then shared wavelength conversion. This difference bring several orders of magnitude enhancement in $B P$ performance, especially for the case with a small number of system wavelengths, offering a practical deployment scenario of 'pay-as-you-grow'. 


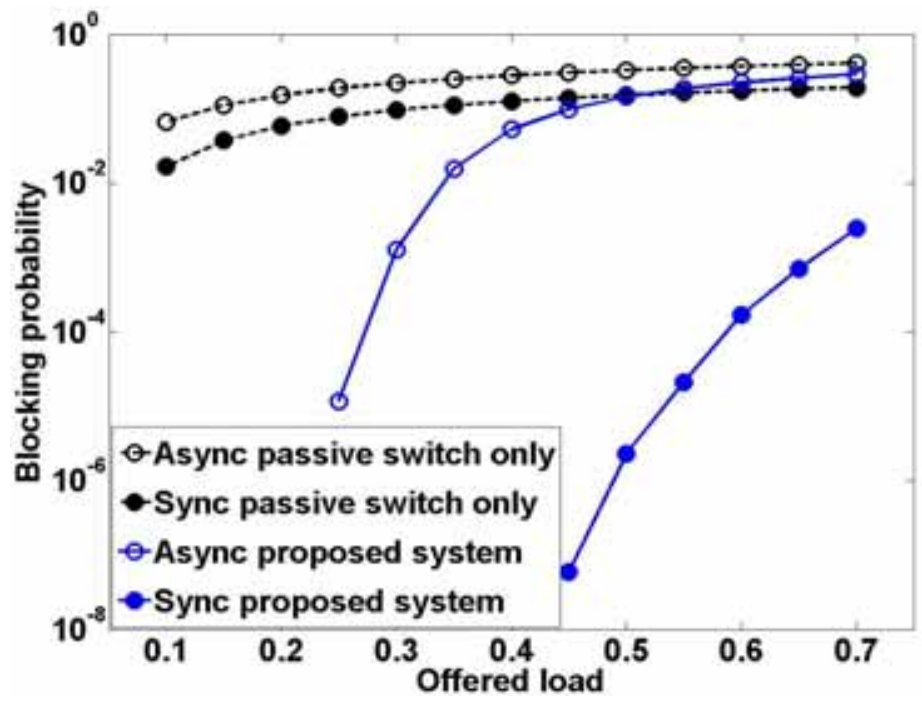

Fig. 2. Blocking probability versus offered load. The number of system wavelengths is 32 and the buffer sharing ratio is $15 \%$. The proposed time-slotted ("sync") system shows orders of magnitude improvements.

\section{Performance and cost analysis}

The benefit of the proposed system is estimated by computer simulation. The blocking performance and system cost are found under the following system design parameters: the number of input/output fiber links $(F)$ is 8 , per-wavelength data rate $10 \mathrm{Gbps}$, and the average optical packets size 100kbyte. These typical design parameters [5] require optical switches with sub microsecond response time. Optical packets are assumed to be uniformly distributed to all output fiber links. The electrical buffer is assumed to have a large enough depth of buffering, so there is no buffer overflow. Fig. 3 presents the sharing ratio requirements for the shared OEO buffers as a function of an offered load in comparison between asynchronous and time-slotted synchronous cases. Two different time-slotted OPS/OBS systems are considered: a fixed-size synchronous packet $\left(\mathrm{N}_{\max }=1\right)$, and a bi-step variable-size synchronous packet $\left(\mathrm{N}_{\max }=2\right)$. Astonishingly, the time-slotted case requires a sharing ratio of only $12 \%$ in the case of 64 wavelength system, in order to provision for an offered load of 0.5 . This is almost a factor of 10 improvement in the OEO requirement with respect to a current electrical cross connect (EXC) system. 


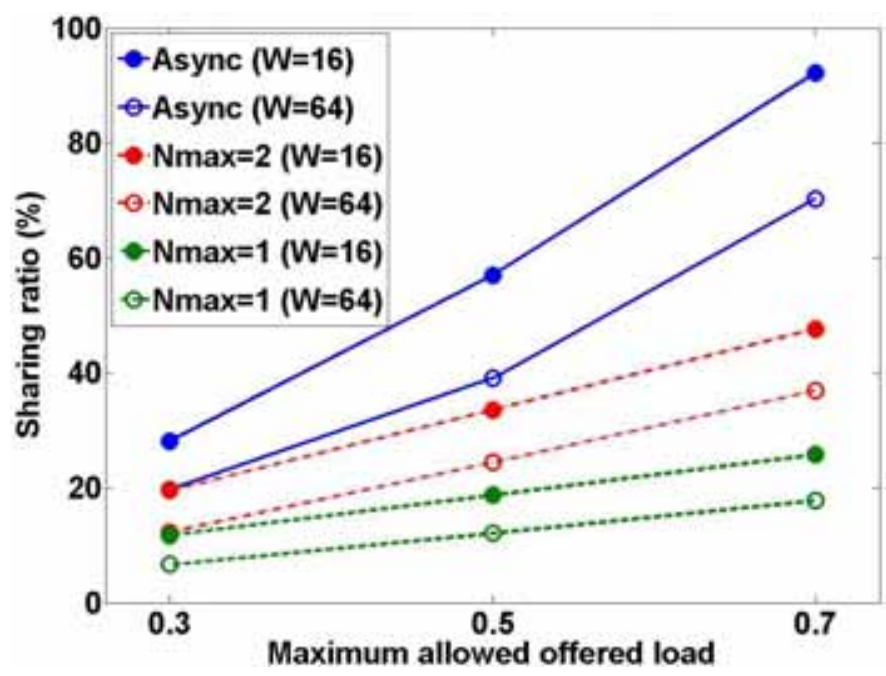

Fig. 3. OEO buffer sharing ratio requirement for blocking probability less than $10^{-6}$ for various conditions. $\mathrm{N}_{\max }=1$ and 2 correspond to fixed-size and bi-step-size time-slotted switching systems, respectively.

Fig. 4 shows overall system cost comparisons of the proposed system with WSSPB [6] and EXC systems for the same performance. The system cost of the proposed system is only $28 \%$ of the traditional EXC cost. Interestingly, the overall cost of the synchronous system is much less than that of asynchronous system.

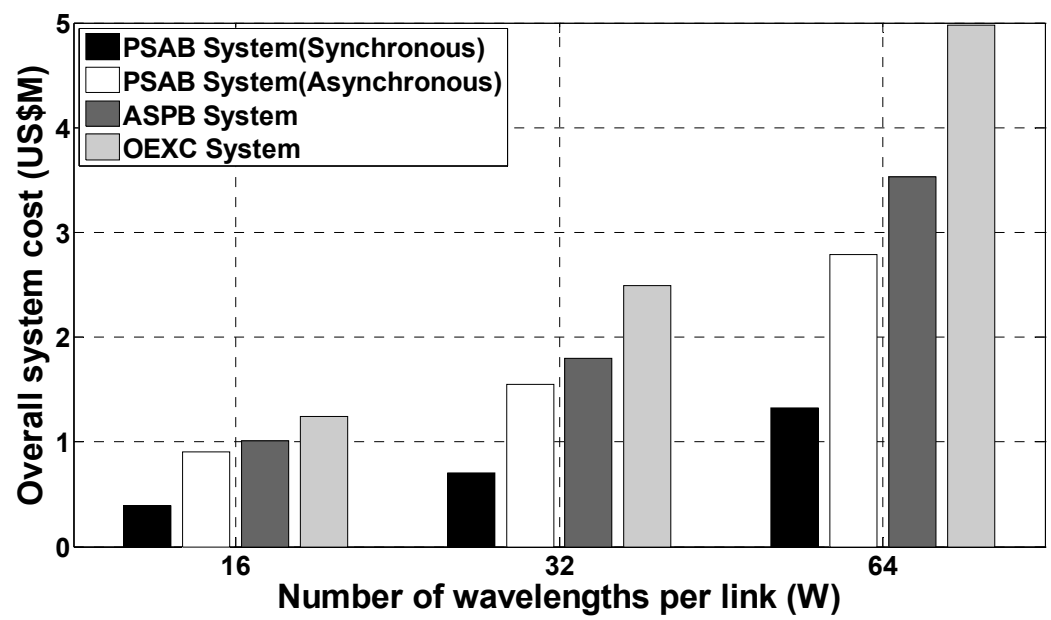

Fig. 4. Overall system cost comparison with constraint of target blocking probability at $10^{-6}$ 
Packet (or burst) switched DWDM networks require packet (or data burst) forwarding to the destined output fiber of a node with or without conversion of the wavelength. When only a fast passive switch fabric is used, the availability of the output fiber is limited to that of Aloha network because of no wavelength conversion. By the theory, several tens of percentiles

\section{Conclusion}

We proposed a novel passive optical switching system with shared OEO packet buffers that may open a new paradigm for OPS/OBS practical applications. The use of shared electrical buffer can achieve several orders of magnitude improvement in blocking probability and potentially require very low power consumption. With practical system design parameters, only $12 \%$ of OEO buffers with respect to the total channels are required to achieve a $10^{-6}$ node blocking probability.

Acknowledgments. This work was supported in part by the KOSEF-OIRC project.

\section{References}

1. A. Bianco, E. Leonardi, M. Munafo, F. Neri, and W. Picco, "Design of optical packet switching networks," Proceedings of GLOBECOM '02, vol. 3, pp. 2752-2756 (2002).

2. S. Verma, H. Chaskar, and R.Ravikanth, "Optical burst switching: a viable solution for terabit IP backbone,” IEEE Network, vol. 14, no. 6, pp. 48-53 (2000).

3. C. Develder, M. Pickavet, and P. Demeester, "Choosing an appropriate buffer strategy for an optical packet switch with a feed-back FDL buffer," Proceedings of ECOC '02, vol. 3, pp. 1-2 (2002).

4. C. M. Gauger, "Optimized Combination of Converter Pools and FDL Buffers for Contention Resolution in Optical Burst Switching," Photonic Network Communications, vol. 8, no. 2, pp. 139-148 (2004).

5. P. Bayvel and M. Dueser, "Optical burst switching: research and applications," Proceedings of OFC '04, vol. 2, pp. 4 (2004).

6. Ji-Hwan Kim, JungYul Choi, Minho Kang, and J.-K. Kevin Rhee, "Design of novel passive optical switching system using shared wavelength conversion with electrical buffer," IEICE Electronics Express, to be published in the issue of December 25, 2006. 